\title{
6 Systemische Therapie mit jugendlichen Sexualstraftätern in einer symptomhomogenen Gruppe
}

Wilhelm Rotthaus

\subsection{Einführung}

Nicht zuletzt durch das „Gesetz der Bekämpfung von Sozialdelikten und anderen gefährlichen Straftaten “ (s. Hammerschlag und Schwarz 1998) ist in der letzten Zeit eine lebhafte Diskussion über Möglichkeiten und Grenzen, Formen und Beurteilungskriterien einer Therapie von Sexualstraftätern aufgekommen. Im Folgenden wird über langjährige Erfahrungen in der stationären Therapie mit jugendlichen und heranwachsenden Sexualstraftätern berichtet. Es werden die Aspekte Aufnahmekriterien in die stationäre Behandlung, Vorteile symptomhomogener Wohn- und Therapiegruppen, Notwendigkeit systemischer Familienarbeit sowie Kriterien für eine Veränderungseinschätzung als Grundlage für eine prognostische Beurteilung dargestellt.

Therapeutische Konzepte müssen sowohl unter Berücksichtigung der spezifischen Charakteristika der jeweiligen Klientel als auch unter Respektierung der jeweiligen Kontextbedingungen entwickelt werden. Sie sind deshalb nur dann verstehbar und im Hinblick auf ihre Übertragbarkeit in andere Arbeitsfelder zu nutzen, wenn diese Kontextbedingungen bekannt sind.

Im Hinblick auf die nachfolgende Darstellung ist deshalb darauf zu verweisen, dass über die Arbeit mit Jugendlichen und jungen Heranwachsenden berich- 
6 Systemische Therapie mit jugendlichen Sexualstraftätern

in einer symptomhomogenen Gruppe

tet wird, die in einem Gerichtsverfahren wegen ihrer Sexualstraftat für schuldfähig angesehen und zu einer Freiheitsstrafe verurteilt wurden. Die Freiheitsstrafe wurde mit der Auflage zur Bewährung ausgesetzt, sich in die stationäre Behandlung bei uns zu begeben. Minimale Voraussetzung ist ein staatsanwaltschaftliches Ermittlungsverfahren und unsere Erwartung einer entsprechenden richterlichen Entscheidung in der Hauptverhandlung. Unter diesen Voraussetzungen wird die Therapie in einem offenen Haus durchgeführt. Die Jugendlichen müssen bereit sein, die von uns über viele Monate verhängten Ausgangsbeschränkungen einzuhalten; bei gravierender Regelübertretung oder Rückfallverhalten erfolgt die Entlassung und zumeist die Aufhebung der Bewährung mit anschließender Inhaftierung. Die Selbstverständlichkeit dieser Konsequenz ist wichtiger Bestandteil des Konzepts. Sie wird nur möglich durch ein intensives Bemühen um eine gute Zusammenarbeit mit der Justiz.

Der weitere Kontext ist eine kinder- und jugendpsychiatrische Klinik, in der bereits seit 25 Jahren der Behandlung von jugendlichen Sexualstraftätern besondere Aufmerksamkeit gewidmet wird. Diese Therapie war ursprünglich einzelpsychotherapeutisch orientiert; die Jugendlichen und Heranwachsenden lebten in einer Cruppe mit anderen Jugendlichen zusammen, die wegen andersartiger Problemverhaltensweisen behandelt wurden. Das Gruppenmilieu entsprach dem einer üblichen jugendpsychiatrischen Station(vgl. Alves 1990).

Etwa vor zehn Jahren wurde dieses Konzept geändert. Die Behandlung erfolgt seitdem in einer Gruppe von zehn jugendlichen Sexualstraftätern, in der das gruppentherapeutische Milieu sehr spezifisch auf die Besonderheiten dieser Klientel ausgerichtet ist. Angeschlossen ist eine so genannte „Schleusengruppe“ mit fünf Plätzen, die die Jugendlichen/Heranwachsenden nach Beendigung der etwa ein Jahr dauernden Therapiephase (in Kostenträgerschaft der Krankenkasse) für noch etwa ein halbes Jahr (in Kostenträgerschaft der Jugendhilfe) durchlaufen, bei hoher Freizügigkeit und beruflicher Außenorientierung, allerdings unter Betreuung durch im Umgang mit den speziellen Problemen dieser Jugendlichen erfahrene Betreuerinnen und Betreuer. Nach unseren katamnestischen Untersuchungen haben wir die Effizienz unserer therapeutischen Arbeit durch die Zusammenfassung der Sexualstraftäter in einer symptomhomogenen Gruppe wesentlich steigern können. Aber selbstverständlich können auch wir keine langfristige Garantie für die Jahre nach der Behandlungszeit unter von uns nicht mehr zu beeinflussenden Kontextbedingungen geben, wie wir das in einem Fall in sehr dramatischer Weise erfahren mussten. ${ }^{4}$

4 So vergewaltigten zwei ehemalige Patienten eineinhalb bzw. zwei Jahre nach ihrer Entlassung während ihrer Wohnbetreuung im Einzugsbereich der Klinik gemeinschaftlich die Mitbewohnerin der Wohngruppe eines der jungen Männer und ermordeten sie anschließend zur Verdeckung ihrer Straftat. Beide waren von uns mit einer günstigen Prognose in Bezug auf das Risiko weiterer Sexualstraftaten entlassen worden. 


\subsection{Kriterien für die Aufnahme in die Täterbehandlung}

\subsubsection{Formale Kriterien}

Eine Beschreibung der Aufnahmekriterien muss zwischen formalen und inhaltlichen Kriterien unterscheiden, wobei die formalen Kriterien den Kontext für die inhaltliche Arbeit definieren. Eine grundsätzliche formale Voraussetzung ist der juristische Rahmen, wie er oben skizziert wurde. Dieser juristische Rahmen hat drei Funktionen:

1. Er verschafft uns einen Auftraggeber für die angestrebte Behandlung: die Justiz. Sie übernimmt die Verantwortung für die notwendigen Kontrollmaßnahmen. Dies kann auf verschiedene Arten geschehen, denn das Jugendstrafrecht erlaubt unterschiedliche Konstruktionen, die für einen Aufenthalt des Jugendlichen bei uns in der notwendigen Länge im Durchschnitt 1 Jahr - sorgen. Es entsteht ein sog. „Zwangskontext“. Versuche von uns, ohne einen solchen „starken“ Auftraggeber mit jugendlichen Sexualstraftätern zu arbeiten, sind daran gescheitert, dass andere mögliche Auftraggeber, wie z.B. Familien oder Jugendämter, aus unterschiedlichen Gründen den notwendigen Druck für eine Behandlung nicht bis zum erfolgreichen Abschluss aufrechterhalten konnten. Es kam in allen Fällen zu vorzeitigen Abbrüchen.

2. Der juristische Rahmen mit der Androhung von möglichen Sanktionen wirkt auf die Jugendlichen selbst in der Regel sehr motivierend in Bezug auf die Einhaltung unserer Grenzsetzungen vor allem hinsichtlich ihrer Freizügigkeit. Aus Gründen des Opfer-Schutzes müssen die Jugendlichen bereit sein, in unserem offenen Haus zunächst längere Zeit auf Ausgang ohne Begleitung zu verzichten. Dies ist eine der Grundbedingungen für die mögliche Aufnahmezusage. Wer diese Bedingungen nicht respektiert, wird von uns entlassen, und die entsprechenden juristischen Instanzen erhalten unverzüglich Mitteilung.

3. Hier schließt sich die dritte Funktion des juristischen Rahmens an: Durch die Mitteilung über die von dem Jugendlichen provozierte Entlassung ist die Justiz erneut in der Verantwortung zu handeln. Sie muss entscheiden, welche Maßnahmen sie ergreift, um ihren Aufgaben nachzukommen. Diese Funktion, nämlich die Verteilung der Verantwortung für die Durchführung von Straftäterbehandlung auf mehrere Schultern, kann nicht wichtig genug eingeschätzt werden. Jeder, der in diesem Bereich arbeitet, sollte darauf achten, dass alle relevanten gesellschaftlichen Instanzen ihren Teil dieser Verantwortung wahrnehmen. Andernfalls entstehen schnell Situationen, in denen durch Überforderung der an der Therapie Beteiligten eine effektive Behandlung unmöglich wird. Wenn Entweichungen oder erneute sexuelle Übergriffe nicht zur Entlassung führen können, weil keine andere gesellschaftliche Instanz mitverantwortlich ist, kann das die Existenz einer ganzen Therapiegruppe 
6 Systemische Therapie mit jugendlichen Sexualstraftätern

in einer symptomhomogenen Gruppe

gefährden. Entweichungen karikieren den Opferschutz, da niemand wissen kann, was in dieser Zeit passiert. Dasselbe gilt selbstverständlich für jede Art erneuter sexueller Übergriffe (vgl. Gruber 1999).

Weitere formale Voraussetzung für eine Aufnahme ist die Durchführung eines oder mehrerer Vorstellungsgespräche, in deren Verlauf der Jugendliche zu erkennen geben muss, dass er bereit ist, über die ihm vorgeworfenen Straftaten zu sprechen. Anhand der Vorgeschichte und unserem Eindruck hinsichtlich seines Verhaltens wird geklärt, wie eng der eben skizzierte juristische Rahmen angelegt sein muss, um Kooperationsbereitschaft bei dem Jugendlichen zu unterstützen. Zuweilen genügt eine Anzeige; meist ist eine Anklage oder auch eine Verurteilung in einer Hauptverhandlung mit einer Bewährungsauflage zur Behandlung bei uns notwendige Voraussetzung. Gelegentlich ist auch die Verbüßung eines Teiles der Haftstrafe oder von Untersuchungshaft notwendig, um den Jugendlichen zur Einhaltung unserer bereits beschriebenen Grenzsetzungen zu bewegen.

\subsubsection{Inhaltliche Kriterien}

Über Berichte (Arztbriefe, Heimberichte, Gutachten, Gerichtsurteile u.a.) sowie in Vorstellungsgesprächen bemühen wir uns um Informationen, die für die diagnostische Einschätzung des Jugendlichen bedeutsam sind. So interessieren wir uns neben einer detaillierten Schilderung der Sexualdelikte für die erotischen Phantasien des Jugendlichen, beispielsweise für seine OnaniePhantasien. Wir versuchen die Häufigkeit der sexuellen Übergriffe zu erfahren und fragen nach den Methoden, mit denen der Widerstand des Opfers überwunden wurde, insbesondere nach Gewaltanwendung. Ebenfalls relevant ist die Planung, die einem Delikt voranging:

- Handelt es sich um eine Straftat mit längerer Kontaktaufnahme zu einem Opfer, oder wird ein eher ungeplantes, spontanes Vorgehen geschildert?

- Sind (wesentlich?) jüngere Kinder als Opfer gewählt worden oder gleichaltrige Mädchen bzw. Jungen?

Wichtige Aufschlüsse erhalten wir daraus, ob der Jugendliche bereit und in der Lage ist, detailliert über seine Taten zu berichten und wie er seine Darstellung sprachlich ausgestaltet - kommt doch hierin zum Ausdruck, inwieweit der Jugendliche Verantwortung für sein Handeln übernimmt (Gruber 1999). Die Quantität und die Qualität der sozialen Kontakte des Jugendlichen zu Gleichaltrigen geben uns Hinweise auf Persönlichkeitsdimensionen wie Beziehungsfähigkeit und Konfliktbereitschaft. In diesem Zusammenhang interessiert auch, wie ein Jugendlicher seine Interessen innerhalb und außerhalb der für ihn relevanten Bezugssysteme zu vertreten in der Lage ist. Um das Ausmaß möglicher Neurotisierungen durch eigene Missbrauchs- und Gewalterfahrungen einschätzen zu können, fragen wir nach entsprechenden Erfah- 
rungen, wohl wissend, dass wir zu diesem Zeitpunkt hierzu oft nur bruchstückhafte Informationen erhalten.

Eine deutliche ausgeprägte Motivation zur Therapie wird von uns nicht verlangt. Auch leichtere Einschränkungen der intellektuellen Fähigkeiten sind kein Ausschlusskriterium (im Gegenteil bei dieser Klientel überproportional häufig). Problematisch für eine Aufnahme kann jedoch das Vorliegen einer ausgeprägten Minderbegabung bzw. einer geistigen Behinderung sein. Unser Konzept therapeutischer Arbeit verlangt ein Mindestmaß an kognitiven Fähigkeiten, um verbale Auseinandersetzungsfähigkeit, Introspektions- und Empathiefähigkeit erlernen zu können. Schließlich sind erste Eindrücke und Informationen über die aktuelle familiäre Situation oder ggf. den alternativen Lebenskontext des Jugendlichen von Bedeutung, was einen frühen Kontakt zur Familie des Jugendlichen notwendig macht. Denn alle Verhaltensweisen positiver und negativer Art sind immer auch Beziehungsbeschreibungen zu wichtigen Personen des Lebensumfelds. Deshalb sind die Frage nach dem Sinn und der möglichen Funktion des sexuell delinquenten Verhaltens im primären Bezugsfeld und die Erkundung familiärer Ressourcen und Unterstützungsmöglichkeiten wichtige Themenfelder.

Zusammengefasst erlauben diese Vorinformationen eine grobe Einschätzung des sexuell auffälligen Verhaltens und der Persönlichkeit des Jugendlichen. Häufig sehen wir Jugendliche mit einer sozial inkompetenten, regressiven und auch fixiert pädophilien Ausrichtung. Etwa gleich häufig werden uns sozial aggressive Jugendliche vorgestellt, die neben den sexuellen Auffälligkeiten auch anderes grenzüberschreitendes Verhalten zeigen. Bei ihnen ist es notwendig einzuschätzen, ob sie einigermaßen sicher die alltäglichen für uns wichtigen Regeln einhalten werden. Anderenfalls würde die Gefahr bestehen, dass durch ständige Regelverletzungen die therapeutische Arbeit gegenüber pädagogischen Sanktionen gänzlich ins Hintertreffen geriete. Im Extremfall kann eine solche Situation die Erfüllung des therapeutischen Auftrags komplett unmöglich machen.

Stark durch körperliche Gewalt gekennzeichnete sexuelle Übergriffe mahnen uns zur Vorsicht: Zum einen ist ein hohes Maß an körperlicher Gewalt bei der Deliktausführung generell ein Hinweis auf eine schlechte Prognose (Rösler 1997). Zum anderen ist die Dauer des Therapieprozesses zu berücksichtigen, die wir anbieten können. Entsteht der Eindruck, dass dieser Zeitraum (11,6 Jahre) zu kurz sein könnte, müssen wir eine Aufnahme ablehnen. Dabei spielt die Einschätzung der Ressourcen sowohl des Jugendlichen als auch seines Bezugssystems eine wesentliche Rolle.

\subsection{Symptomhomogene Wohngruppen}

Wird die Literatur zum Thema betrachtet, so scheint sich dort unsere Erfahrung bestätigt zu finden, dass die Unterbringung der Täter in symptomhomo- 
6 Systemische Therapie mit jugendlichen Sexualstraftätern

in einer symptomhomogenen Gruppe

genen Wohngruppen (im klinischen Sprachgebrauch: Stationsgruppen), d.h. die Bildung von Gruppen, in denen ausschließlich Sexualstraftäter zusammenleben, das Mittel der Wahl ist (Wiederholt 1989; Thomas-März u. MüllerIsberner 1995; Schmidt 1996). Im Rückblick auf zwei Jahrzehnte Tätertherapie in unserer Klinik sind wir überzeugt, die Effektivität unserer Arbeit vor allem dadurch gesteigert zu haben, dass wir eine symptomhomogene Gruppe mit sexualdelinquenten Jugendlichen bildeten.

In Übereinstimmung mit Schmidt (1996: 6) hatten wir die Erfahrung machen müssen, dass in symptomheterogenen Cruppen sexualdelinquente Jugendliche in ihrer therapeutisch extrem kontraproduktiven Neigung verstärkt werden, ihr Delikt zu verheimlichen, zu bagatellisieren und zu leugnen. Denn bei Bekanntwerden des Delikts bestand die Gefahr, von den anderen Mitbewohnern zumindest ausgeschlossen, verachtet und gedemütigt zu werden. In der Delikthierarchie stehen bekanntlich - auch in Haftanstalten - Sexualstraftäter auf der untersten Ebene und sehen sich mancherlei Misshandlungen ausgeliefert, nicht zuletzt solchen sexueller Art, was die Rückfallgefahr des Täters erhöht.

In symptomhomogenen Tätergruppen demgegenüber wird das möglich, was als oberstes Prinzip therapeutisch/pädagogischer Arbeit mit Sexualstraftätern zu gelten hat: Offenheit. So müssen bei uns alle Jugendlichen über Art, Anzahl und Ausführung der Delikte, die sie begangen haben, bis in alle Einzelheiten vor der Gruppe und mit Unterstützung der Gruppe berichten. Hilfreich sind in diesem Zusammenhang Aufnahme- oder Eröffnungsrituale, die jedes neue Gruppenmitglied dazu verpflichten, am Abend des Aufnahmetages vor der Gruppe genau seine Übergriffe zu schildern. Die schon länger anwesenden Gruppenmitglieder tun - jedes Mal erneut dasselbe und zeigen damit, dass sie gelernt haben, über ihre einschlägigen Taten zu sprechen und sie als Teil ihrer persönlichen Geschichte zu bekennen. Des Weiteren haben sich Gesprächsrunden bewährt, in denen die neuen Gruppenmitglieder durch die schon länger Anwesenden peinlich genau zu ihren Delikten befragt werden. Hier geht es um die Häufigkeit von Delikten, das Einräumen bisher nicht bekannt gewordener Straftaten, um das Ausmaß an Gewaltanwendung etc. Die Cruppenmitglieder befragen oft schonungsloser, als es in der Einzeltherapie möglich ist, und kennen die typischen Abläufe bestens.

Generell sind wir bestrebt, die Stations- bzw. Wohngruppe als therapeutische Gemeinschaft wirksam werden zu lassen. Dieser Ansatz beruht „auf der Idee und dem Vertrauen, dass eine subtile Steuerung einer Zwangsgemeinschaft mit gruppendynamischen Methoden und praktizierte Offenheit in dieser Gemeinschaft möglich sei und nicht nur momentane, sondern dauernde Veränderungen in Richtung prosozialer Einstellungen und Verhalten, somit, Besserung" bewirkt". Rotthaus (1990) erörtert eingehend die Anwendung der Grundsätze und Prinzipien der therapeutischen Gemeinschaft auf die Arbeit in einer jugendpsychiatrischen Klinik. Hilpert und Schwarz (1981: 1611) folgend 
hebt er u.a. hervor: Soll eine Institution zu einem sozialen Organismus mit therapeutischer Funktion entwickelt werden, sind hierarchische Strukturen durch horizontale, demokratische zu ersetzen. Entscheidungsbefugnisse müssen soweit wie möglich delegiert werden. Durch eine klare und transparente Trennung von Verantwortungsbereichen wird ein Höchstmaß an Eigenverantwortlichkeit einer jeden Mitarbeiterin und eines jeden Mitarbeiters erreicht. Leitende Mitarbeiterinnen und Mitarbeiter haben eine Steuerungsfunktion, die in der Beurteilung besteht, ob Freiräume kompetent genutzt werden und das Handeln der Erfüllung des Gesamtziels dient. Die Patienten übernehmen selbstverantwortlich wichtige Funktionen in der Cestaltung des Zusammenlebens. Die Zweiteilung in Behandelnde und Behandelte wird relativiert und in ihrer strikten Form als hinderlich für den therapeutischen Prozess betrachtet. Die Patienten übernehmen für sich und für andere therapeutische Funktionen.

Ein solches Konzept erfordert eine Durchlässigkeit der Kommunikation über alle Ebenen sowie offenen Austausch von Informationen und Gefühlen. Um eine Analyse der Kommunikation zu ermöglichen, muss Toleranz gegenüber den Äußerungen jeden einzelnen Mitglieds einer Behandlungsgemeinschaft gewahrt werden. Regelmäßige Reflexionen der Vorgänge in einer solchen Gemeinschaft sind erforderlich, beispielsweise auf Stationsversammlungen. Die Offenheit eines solchen Systems für Kommunikationen mit der Umwelt ist ebenso erforderlich. Die Einbeziehung der Angehörigen in den therapeutischen Prozess sowie Arbeitsversuche außerhalb und Beurlaubungen gehören somit zum Programm. In der Konsequenz bedeutet dies, dass eine Einrichtung dann zu einer therapeutischen Institution wird, „wenn es gelingt, ein multipersonales Beziehungsangebot zu verwirklichen, dass die Möglichkeit zu therapeutischen Entwicklungserfahrungen auf den verschiedensten Ebenen bietet, mit anderen Worten, wenn ein Kontext - und zwar nicht nur für Patienten, sondern auch für Angehörige (!) - geschaffen wird, der zu erneuter Koevolution herausfordert“ (Rotthaus 1990: 162).

\subsection{Systemische Gruppenarbeit}

Während das Konzept der therapeutischen Gemeinschaft dazu dient, ein therapeutisch wirksames, wenn auch vorwiegend pädagogisch beeinflusstes Alltagsleben zu realisieren, sind mit „Gruppentherapie“ zeitlich begrenzte Kontexte zur gezielten Bearbeitung umschriebener Anliegen und Themen angesprochen. Wer z.B. „Offenheit“ in der oben beschriebenen Weise herstellen will, kann dies nicht besser tun, als durch Arbeit in therapeutischen Gruppen. Gruppentherapie bietet in hervorragender Weise die Möglichkeit, die Gefühle der anderen Cruppenmitglieder, die als Reaktion auf die Äußerung eines Jugendlichen entstehen, zu nutzen. Soziale Bezogenheit von Verhaltensweisen, die sonst geheim und verdeckt ablaufen und deren Reflektion und Offen- 
6 Systemische Therapie mit jugendlichen Sexualstraftätern

in einer symptomhomogenen Gruppe

legung verboten ist, kann auf diese Weise schnell und effektiv deutlich gemacht werden. Für die anderen Gruppenmitglieder ist in dieser Situation stellvertretendes Lernen möglich. Jeder kann Parallelen zwischen sich und dem Gegenüber ziehen und auf seine Weise verarbeiten.

Gruppentherapie wird in unserer Einrichtung von unterschiedlichen Professionen, in unterschiedlichen Zusammensetzungen, mit unterschiedlichen thematischen Schwerpunkten und mit unterschiedlichen methodischen Ausrichtungen - wenn auch durchweg unter einer übergeordneten systemischen Orientierung - durchgeführt. So gibt es eine Delikt orientierte Tätergruppe, die vom leitenden Dipl.-Psychologen und einer erfahrenen Ärztin geleitet wird. An ihr nehmen alle Jugendlichen der Stations- bzw. Wohngruppe teil. Dies gilt auch für die Gruppe „Billy Boy“, die von den Gruppenbetreuerinnen (Erzieherinnen/Erzieher bzw. Krankenschwestern und -pflegern) durchgeführt wird. Der thematische Schwerpunkt dieser Gruppe kann mit sexueller Aufklärung und antisexistischer Jungenarbeit umschrieben werden. Zwei weitere Gruppen sind ebenfalls obligatorisch: Von einer stationsübergreifend tätigen Sozialpädagogin wird die sog. ,Jugendkonferenz“ betreut, in der die Jugendlichen alltagsorientierte Themen ihres Zusammenlebens besprechen (und die deshalb richtiger der als therapeutische Gemeinschaftskonzipierte Wohngruppe zuzuordnen ist). Hinzu kommt eine Kunsttherapiegruppe. Diese Arbeit erfolgt themenzentriert und soll die Jugendlichen anregen, inneres Erleben in bildnerischem Gestalten auszudrücken und anschließend im Kreis der anderen zu reflektieren. Im Übrigen besuchen die Jugendlichen je nach von uns eingeschätztem Bedarf und eigenem Interesse auf freiwilliger Basis die in der Klinik stationsübergreifend angebotenen Gruppen zum Training sozialer Interaktionskompetenzen sowie das in seiner sozialtherapeutischen Bedeutung nicht zu unterschätzende von der Klinik organisierte Freizeitzentrum.

Im Folgenden seien noch einige Merkmale der Delikt-orientierten Tätergruppe geschildert, die verständlicherweise einen zentralen Stellenwert hat. Es handelt sich um eine offene, fortlaufende Gruppe, die es dem neu hinzugekommenen Täter ermöglicht, andere Täter zu erleben, die offen, mit angemessenen Worten und mit angemessener emotionaler Beteiligung über ihre Delikte sprechen. Dies kann Anstoß sein, die eigenen Verleugnungen aufzugeben. Der Täter erlebt andere Täter, die ihm signalisieren, dass er ohne Verleugnung existieren kann, dass die Funktion seiner Verleugnung, ihn zu schützen, überflüssig geworden ist, dass „Experten“ ihm erlauben, auch Anteile von Gewalt und Sadismus bzw. in anderer Weise Schambesetztes zuzugeben, und ihm versichern, dass er auch nach solchen Eröffnungen weiter existieren kann und von den übrigen Gruppenmitgliedern akzeptiert wird. Er macht sogar die Erfahrung, dass er mit dieser offeneren Haltung besser in der Cruppe zurechtkommen wird.

Weitere Aspekte, die auch andere Autoren (Hendriks und Bullens 1998) aufführen, sind: Das offene Sprechen vor anderen Menschen über die eigenen 
Delikte ist bereits ein erster Schritt zur Übernahme von Verantwortung. Der Täter kann lernen, eine andere Sprache zu erwerben, die durch Verantwortlichkeit statt durch Verantwortungslosigkeit gekennzeichnet ist.

Mitglieder einer offenen Gruppe können ihr eigenes Tempo wählen. Sie haben Zeit, die anderen zu beobachten, Mut zu fassen und nach und nach in die Gruppe hineinzukommen. So wird kein unnötiger Widerstand provoziert und die Gesamtgruppenaktivität, die meist von den erfahrenen Gruppenmitgliedern bestimmt wird, leidet darunter nicht. Das aktuelle, auf andere bezogene Verhalten kann in der Gruppensituation thematisiert und therapeutisch genutzt werden. Bezüge zum Deliktverhalten sind oft möglich, vor allem dann, wenn es in der Gruppe auch potenzielle Opfer gibt.

In der Tätergruppe wird der Täter wahrscheinlich erstmalig sehr deutlich mit den Gefühlen konfrontiert, die sein Deliktverhalten bei anderen auslöst: Gefühle von Ekel, von Wut, von Abscheu und oft von Mitleid mit dem Opfer sind die Regel. Hier ist Offenheit auf Seiten der Therapeuten gefordert und unverzichtbar, wenn diese handlungsfähig bleiben wollen. Würden wir solche Gefühle gegenüber dem Täter verschweigen, würde das einem wichtigen therapeutischen Ziel, nämlich seine Empathiefähigkeit zu wecken und zu stärken, zuwiderlaufen. Die Konfrontation mit den Gefühlen, die seine Tat(en) bei anderen auslösen, wird er im Laufe des Aufenthaltes noch häufig - nicht zuletzt in der Begegnung mit den Betreuerinnen und Betreuern der Gruppe - durchstehen müssen; es wird ihm gelingen, wenn er bemerkt, dass sein Verhalten und nicht seine Person gemeint ist. An diesem Punkt zeigt sich eine wichtige, sowohl für das therapeutische Team als auch für den Täter nicht einfache Dialektik: Einerseits muss dem Täter vermittelt werden, dass seine Tat(en) mit seiner Person zu tun haben und er die persönliche Verantwortung für sie und vor allem für sein zukünftiges Verhalten zu übernehmen hat. Andererseits ist die Botschaft wichtig, dass seine Tat(en) bei den Mitgliedern des therapeutischen Teams zwar Betroffenheit, Wut und auch Abscheu - in individuell und geschlechtsspezifisch unterschiedlicher Ausprägung - auslösen, dass er als Person aber akzeptiert und wert geschätzt wird.

Aus den Niederlanden (vor allem: Hendriks und Bullens 1998) wird berichtet, dass sich dort vorgefertigte Module in der Täterarbeit bewährt haben, die in einer festgelegten Abfolge durchgearbeitet werden. Dem steht nach unseren Erfahrungen das Primat des aktuell von der Gruppe formulierten Themas und der Beziehung zwischen Täter(n) und Therapeuten entgegen (s.a. Barnes 1998). Auch transportiert der Modulaufbau allzu leicht die Idee, mit der Absolvierung eines Moduls durch den Täter sei das Thema für ihn umfassend abgehandelt und mit dem Durchlaufen eines solchen, aus Modulen zusammengesetzten Programms sei mehr oder weniger zwingend eine gute prognostische Einschätzung verbunden. Vorgefertigte Strukturelemente machen den Behandlungsprozess für den Täter sicherlich überschaubarer. Sie bergen jedoch gerade in ihrer Transparenz die Gefahr, dass die häufig sehr gut angepassten Täter 
6 Systemische Therapie mit jugendlichen Sexualstraftätern

in einer symptomhomogenen Gruppe

sich an die immanent formulierten Erfolgskriterien anpassen und somit Veränderungen vortäuschen (s.a. Müller-Isberner u. Thomas 1992). Wir denken, dass ein guter Teil Unberechenbarkeit immer notwendig ist. Therapie bedeutet Verunsicherung. Sie darf nicht zu sehr reduziert werden; andernfalls wird dem Täter letztendlich die Chance zur Veränderung genommen.

\subsection{Systemische Familienarbeit}

Mit O'Callaghan u. Print (1994) und Barnes (1998) sind wir der Meinung, dass systemische Familienarbeit unverzichtbarer Bestandteil der Arbeit mit jugendlichen Sexualstraftätern ist. Als Begründung für diese Forderung führt Barnes aus, dass die Familie die Verhaltensweisen, Einstellungen, Überzeugungen und Wertsysteme des Täters beeinflusse und forme, und zwar weitgehend unabhängig davon, ob der Täter in der Familie lebe oder nicht. Durch den Einbezug der Familie in das Täterprogramm könne man die familiären Einflüsse explorieren, die zur Tatentstehung beigetragen hätten. Allerdings sei dies nicht ein Versuch, die Verantwortlichkeit für die Tat auf irgendjemand anders als den Täter zu lenken. Es gebe keinen Anlass anzunehmen, dass familiäre Faktoren wie eingeschränkte Kommunikation, unklare Grenzen und Beziehungsabbrüche kausale Faktoren für Sexualstraftaten seien, auch wenn sie möglicherweise zu einem Klima beigetragen hätten, die den Jugendlichen für bestimmte Arten von Täterverhalten sensibilisiert hätten. Familienarbeit sei aber vor allem Präventionsarbeit; denn Familienmitglieder spielten meist eine wichtige Rolle in der oft wenig strukturierten Zeit nach der Behandlung.

Wie der Täter nicht sofort ein Gefühl für seine Schuld entwickelt, so könne auch die Familie eine derartig dramatische Eröffnung zunächst schwer akzeptieren und neige zu Verleugnungen, Verharmlosungen und Entschuldigungen. Häufig werde dies zusätzlich durch Familiengeheimnisse erschwert, die dazu dienten, den familiären Zusammenhalt zu wahren. Familienarbeit gebe die Möglichkeit, sexuelle Verantwortlichkeit in das Familiensystem zurückzubringen, was häufig dazu beitrage, dass frühere Opfererfahrungen einzelner Familienmitglieder ans Tageslicht treten (Barnes 1998: 118).

Die von uns konstruierten Kontextbedingungen, die weiter vorne geschildert wurden, haben zur Folge, dass nicht die Eltern Auftraggeber für die Behandlung des Jugendlichen sind, sondern vielmehr ,gesellschaftliche Institutionen“. Diese nehmen den Eltern mit ihrer Auftragserteilung die Möglichkeit, frei und ohne Einschränkungen den Aufenthalt ihres Kindes zu bestimmen. Elterliche Verantwortlichkeit wird ersetzt durch die Verantwortlichkeit juristischer Instanzen. Die Eltern werden „entmündigt“, und diese Entmündigung verstärkt die fast durchweg zu beobachtende familiäre Tendenz, das Delikt des Jugendlichen zu leugnen, $z u$ bagatellisieren, $z u$ entschuldigen oder ihm mit völliger Sprachlosigkeit zu begegnen. Dies führt zu einem Rückzug des 
elterlichen Bezugssystems von therapeutischer Mitarbeit, wenn nicht in angemessener Weise auf diese Situation reagiert wird.

Barrett und Trepper (1997) beschreiben Strategien, die den therapeutischen Umgang mit diesen Systemen ermöglichen. Kernstück ist, das Systemverhalten als sinnvolle Haltung des Systems zu akzeptieren, sinnvoll beispielsweise dafür, das System überhaupt „am Leben zu erhalten“. Nach unseren Erfahrungen ist es möglich, mit dieser Haltung betroffenen Systemen die Zeit zuzugestehen, bis eine Thematisierung der relevanten Themen möglich ist. So finden mit den Bezugssystemen unserer Jugendlichen zu Beginn eines Aufenthaltes zwar Vorgespräche und Eingangsgespräche statt, im weiteren Verlauf sehen wir die Familien unserer Jugendlichen aber eher selten. Diese sind häufig auch nur an Berichten über die Entwicklung des Jugendlichen interessiert, die wir ihnen bereitwillig geben. Unsererseits zeigen wir Interesse an der familiären Entwicklung, ohne jedoch auf „Therapie“ zu drängen (Layne 1994).

Diese Haltung ermöglicht in den meisten Fällen wachsende Offenheit und Bereitschaft der Familien, sich auf ein therapeutisches Bündnis einzulassen. Häufig verläuft dies parallel zur Entwicklung des Jugendlichen, wobei die Jugendlichen manchmal sogar die „Schrittmacher“ für die Entwicklung in der Familie sind: Beginnende Offenheit beim Jugendlichen löst in der Familie Leugnungen auf und vermindert Bagatellisierungen, was wiederum beim Jugendlichen zu größeren Entwicklungsschritten führen kann. Je näher die Entlassung kommt, umso intensiver gestalten wir die Arbeit mit der Familie. Dies hängt selbstverständlich von der Frage ab, ob der Täter in seine Familie zurückkehrt. Allerdings stehen der Rückkehr des Täters in die Familie zuweilen unüberwindliche Schwierigkeiten gegenüber. Oft ist dies nur möglich, wenn die Familie zu einem Umzug bereit und in der Lage ist. Die Stigmatisierung des Jugendlichen durch seine Tat(en) ist in der alten häuslichen Umgebung meist so hoch, dass dort eine Reintegration in Schule und Beruf kaum möglich ist.

Anders stellt sich die Situation dar, wenn wir es mit einer innerfamiliären sexuellen Misshandlung zu tun haben. Auch hier beobachten wir nach anfänglich zumeist heftiger Reaktion des Systems, die in einer Ausstoßung des Jugendlichen gipfeln kann, sehr bald die bereits beschriebenen Leugnungsund Bagatellisierungstendenzen. Bei innerfamiliären sexuellen Misshandlungen erscheint es uns sinnvoll und notwendig, möglichst schnell mit systemischer Familienarbeit zu beginnen, die dann auch in häufiger Frequenz stattfindet. Hierbei hat es sich bewährt, sich an dem von Eddy (1991) und Madanes (1997) entwickelten Vorgehen zu orientieren, insbesondere wenn eine Rückkehr in die Familie zumindest als möglich erachtet wird. Das von den beiden Autoren beschriebene Vorgehen erscheint uns in der Systematik hilfreich. Wenn auch die von ihnen benannte Stufenfolge des Vorgehens nicht starr eingehalten werden muss, so sind doch die Prinzipien Offenlegen aller 
6 Systemische Therapie mit jugendlichen Sexualstraftätern

in einer symptomhomogenen Gruppe

Delikte, Begründen und Erklären von Art und Ausmaß der Schädigung, Entschuldigung (in welcher Form auch immer), wiedergutmachende Handlungen, Versöhnung (wenn möglich) unverzichtbare Bestandteile.

\subsection{Kriterien für eine Veränderungseinschätzung als Grundlage für eine prognostische Beurteilung}

Das beschriebene therapeutische Setting macht es notwendig, kontinuierlich die Fortschritte der Jugendlichen in der Therapie zu beurteilen, um über das Ausmaß der Fremdkontrolle entscheiden und schließlich eine Prognose bezüglich des weiteren Verhaltens des Jugendlichen abgeben zu können. Wie generell in der Therapie, lassen sich diese Fortschritte an der Entwicklung fort von einer Überzeugung des Ausgeliefert-Seins an die individuelle Geschichte oder an höhere Mächte hin zu Verantwortung und Kontrollüberzeugungen ablesen (siehe: locus-of-control-Forschung, z.B. Krampen 1991).

Diese Entwicklung wird erkennbar an den konkreten Verhaltensweisen des Jugendlichen im zwangsläufig engen Kontakt zu den anderen Tätern in der Cruppe, im weiteren zu den übrigen Jugendlichen (Mädchen wie Jungen) in Schule und Werkstatt sowie zu den Mitarbeiterinnen und Mitarbeitern, zum anderen an den generellen Haltungen des Jugendlichen, vor allem an seiner Einstellung zu den von ihm begangenen Taten und seinen künftigen Verhaltensweisen im Bereich der Sexualität. Darüber hinaus spielen die Entwicklung von Empathieverhalten spezieller und genereller Art sowie die Entwicklung von Kooperationsverhalten eine wichtige Rolle. Retrospektiv muss selbstverständlich auch das Ausmaß von Gewaltanwendung während der Sexualstraftaten bei der Prognose berücksichtigt werden. In regelmäßigen Sitzungen des gesamten pädagogisch-therapeutischen Teams werden die Beobachtungen aller Mitarbeiterinnen und Mitarbeiter über die einzelnen Jugendlichen zusammengetragen, um eine Veränderungseinschätzung vornehmen zu können. Folgende Kriterien für eine solche Bewertung haben sich in unserer Arbeit bewährt. Die Beispielssätze sollen die Entwicklung des Jugendlichen von ... (linke Seite) zu ... (rechte Seite) veranschaulichen (s. Tab. 4). (Die Form der Darstellung wurde in Anlehnung an Russinger u. Wagner [1999] gewählt, von denen auch einige Anregungen aufgenommen wurden.)

\subsection{Abschluss}

Es mag verwundern, dass so wenig über die (systemische) Einzeltherapie mit den Sexualstraftätern unserer Gruppe berichtet wurde. Das hat seinen Grund darin, dass wir Einzelpsychotherapie als eine soziale Aktivität ansehen, die sich dadurch konstituiert, dass eine Person ein Problem bei sich erkennt und diese einen Therapeuten bittet, ihr bei der Lösung dieses Problems behilflich zu 
Tab. 4 Die Entwicklung des Jugendlichen in der Therapie anhand von Beispielsätzen

Beziehung des Jugendlichen zu seinen Delikten
Entwicklung von Opfer-Empathie

„Es tut mir leid, was ich getan habe, weil ich er- „Es tut mir leid, was ich getan habe, weil ich andewischt worden bin und jetzt Probleme habe." ren sehr wehgetan habe."

\section{Kenntnis der eigenen Verführbarkeit}

„In solchen Situationen kann ich nicht anders, das macht mich an, da kommt es über mich."
„Ich kenne genau die für mich schwierigen Situationen und weiß, an welchem Punkt ich mich entscheiden muss auszusteigen."

\section{Grad der Verantwortungsübernahme}

„Eigentlich ist doch nichts passiert.“

„Ich habe mich entschieden, die sexuelle Selbstbestimmung."

\section{Art der Kontrollüberzeugung}

„Es kommt über mich, hat nichts mit mir zu tun." „Ich weiß, was ich in Zukunft tue.“

\section{Beziehung zur Sexualität}

\section{Fähigkeit, Grenzen anderer wahrzunehmen und zu respektieren}

„Die Kinder wollten das auch; sie haben sich gar nicht richtig gewehrt.“
„Nein heißt Nein, und kein Nein ist noch lange kein a."

\section{Verantwortungsübernahme angesichts eigener perverser Fixierungen}

„Ich bin so geboren und kann nichts dafür."

„Ich bin wie ich bin, und habe mich entschieden, niemanden zu schädigen."

\section{Grad der Kontrollüberzeugung angesichts eigener perverser Fixierungen}

„Ich bin wie ich bin, und kann dagegen nichts machen."
„Ich bin wie ich bin und weiß, wie ich leben kann, ohne andere zu schädigen."

\section{Fähigkeit zum sexuell-erotischen Beziehungsaufbau und -erhalt zu Gleichaltrigen}

„Wenn das Mädchen nicht bei der zweiten Begegnung mit mir schläft, habe ich versagt.“
„Ich genieße das allmähliche Kennenlernen und lasse die sexuelle Begegnung sich entwickeln.." „Ich genieße die vielen Schritte in der Vorphase von Petting und Geschlechtsverkehr und bin in der Lage wahrzunehmen, was meine Partnerin/mein Partner möchte."

\section{Emotionalität}

\section{Grad der Differenzierung der Wahrnehmung und der Verbalisierung der eigenen Gefühle}

„Mir geht es immer nur mies, das kommt wie von selbst."
„In solchen Situationen fühle ich mich traurig; ich kann dies akzeptieren oder etwas dagegen tun." 
6 Systemische Therapie mit jugendlichen Sexualstraftätern

in einer symptomhomogenen Gruppe

\section{Emotionalität}

\section{Umgang mit belastenden Gefühlen}

„Wenn es mir mies geht, dann passiert es; dann muss ich dieses Gefühl wieder loswerden, egal wie oder wo."

„Ich kenne Wege und Mittel, mit einer Kränkung umzugehen: mich zu wehren, sie nicht so ernst zu nehmen, Unterstützung und Verständnis anderen zu suchen, u.a."

\section{Umgang mit aggressiven Impulsen}

„Wenn ich wütend bin, brauche ich ein Kind, um mich abzureagieren." - „Wenn ich wütend bin, schlage ich zu."

„Wenn ich wütend bin, kann ich überlegen, worauf ich wütend bin, und entscheiden, wie ich mit meiner Wut angemessen umgehe."

\section{Stand der Identitätsentwicklung}

\section{Fähigkeit, eigene Standpunkte zu vertreten}

„Wenn ich meine Meinung sage, bekomme ich nur Arger; meinen Frust behalte ich lieber für mich."

„Ich kann entscheiden, ob ich meine Meinung sage, ich kann nicht wissen, was dann kommt. Es gibt aber eine gute Chance, dass es mir dann besser geht."

\section{Umgang mit Konflikten und Frustrationen}

„Bei Konflikten halte ich die Klappe und sehe zu, „Wenn es Streit gibt, kann ich mich wehren, ohne dass ich sie möglichst schnell hinter mich bringe." dem anderen Schaden zuzufügen."

\section{Stand der sozialen Entwicklung}

\section{Allgemeine Fähigkeit zum Beziehungsaufbau und -erhalt zu Gleichaltrigen}

„Ich habe viele Freunde, überhaupt keine Freunde, nur Feinde: Keiner mag mich."

„Manche mögen an mir dieses und anderes nicht, ebenso wie ich bei anderen vieles mag, aber auch nicht alles."

\section{Bedeutungsgebung in sozialen Beziehungen}

„Ich habe viele Freunde, überhaupt keine Freunde, nur Feinde: Keiner mag mich."

„Manche mögen an mir diese und anderes nicht, ebenso wie ich bei anderen vieles mag, aber auch nicht alles."

„Wenn ich eine wichtige Beziehung nicht völlig unter Kontrolle habe, werde ich selbst zum Opfer."

„Manchmal bin ich der Bestimmende und manchmal die anderen. Ich finde es gut, wenn das wechselt. Ich lasse mich in meinem Selbstwertgefühl nicht leicht verunsichern."

\section{Kontrollüberzeugungen allgemein}

„Im Grunde genommen ist alles gleich, was ich tue; ich habe sowieso keine Chance."

„Vieles in meinem Leben ist mir wichtig. Wenn ich mich dafür einsetze, habe ich gute Chancen, etwas davon zu erreichen." 
sein. Dies tut der übliche Sexualstraftäter erst relativ spät im Verlaufe des gesamten Behandlungsprozesses. Er muss erst lernen, sein Verhalten mit seiner Person in Beziehung zu setzen, sein inneres Erleben wahrzunehmen und die Chance der Veränderung durch eigenes Bemühen zu erkennen. Der Jugendliche wird zu Beginn der Behandlung darüber informiert, dass er jederzeit zu dem leitenden Psychologen gehen und um Einzeltherapiestunden nachsuchen kann. Tatsächlich sind die Jugendlichen zumeist erst nach einer längeren Zeit soweit - das kann möglicherweise mehrere Monate dauern. Sind sie an diesem Punkt angekommen, dann unterscheidet sich die Einzelpsychotherapie nicht wesentlich von der Einzelpsychotherapie mit anderen Jugendlichen.

Insgesamt wurden seit 1990, als wir begannen, unser Konzept auf die geschilderte Zielgruppe hin schrittweise zu präzisieren, 8o jugendliche Sexualstraftäter bei uns aufgenommen (Stand Ende Juli 1999). In den ersten sechs Jahren, d.h. bis Ende 1996, brachen ca. ein Drittel von ihnen die Behandlung ab oder wurden von uns wegen Regelübertretungen vorzeitig entlassen. In den letzten vier Jahren, in denen wir noch konsequenter auf dem juristischen Rahmen für unsere Arbeit bestanden haben, ist diese Zahl auf ein Viertel zurückgegangen, wobei die Entlassungen unsererseits in etwa konstant blieben, während die Abbrüche seitens der Jugendlichen und ihrer Angehörigen stark zurückgingen. Eine wissenschaftlich fundierte Outcome-Studie über die Jugendlichen, die die Behandlung regulär bis zu einem von uns befürworteten Ende durchliefen, haben wir aufgrund fehlender zeitlicher und finanzieller Ressourcen leider nicht verwirklichen können, jedoch regelmäßige Nachbefragungen durchgeführt. Die Rückmeldungen, die wir über diese Jugendlichen und ihre weitere Entwicklung durch persönliche Gespräche mit den Jugendlichen selbst, durch telefonische Kontakte oder durch Kontakte mit zuständigen Betreuern erhielten, sind als insgesamt positiv zu werten. So erhielten wir lediglich in dem einen, oben erwähnten, sehr dramatischen Fall von einer erneuten Sexualstraftat Kenntnis. Insgesamt haben wir den Eindruck gewonnen, dass die Arbeit mit Sexualstraftätern gerade im frühen Stadium ihrer einschlägigen Entwicklung eine Erfolg versprechende und dankbare Aufgabe ist. Allerdings ist die psychische Belastung aller pädagogisch-therapeutischen Mitarbeiterinnen und Mitarbeiter angesichts der nahezu täglichen Möglichkeit „falscher" Entscheidungen nicht zuletzt auch wegen der durch die Boulevard-Medien aufgeheizten Öffentlichkeit gerade gegenüber diesem Täterkreis nicht zu überschätzen.

\section{Literatur}

Alves I-I (1990) Therapeutische Arbeit mit sexuell auffälligen Jugendlichen. In: Rotthaus W (Hrsg.) Psychotherapie mit Jugendlichen. Dortmund: modernes lernen, S. 212-227.

Barnes C (1998) Systemic Family Work with Adolescent Sex Offenders. Prison Service Journal 118: 24-27.

Barrett M), Trepper TS (1997) Behandlung der Verleugnung in Familien bei sexuellem Kindesmißbrauch. Kontext 28: $146-162$. 
Duffek H (1997) Therapie mit Tätern im Strafvollzug. In: Amann G, Wipplinger R (Hrsg.) Sexueller Mißbrauch. Tübingen (dgvt), S. 586-602.

Eddy DR (1991) Eine Methode, mit Sexualtätern und ihren Opfern zu arbeiten. In: Rotthaus W (Hrsg.) Sexuell deviantes Verhalten Jugendlicher. Dortmund: modernes lernen, S. 152-162.

Gruber T (1999) Über die Arbeit mit jugendlichen Sexualstraftätern in einem Zwangskontext. In: Wodtke-Wernes V (Hrsg.) Nicht wegschauen! Vom Umgang mit Sexualstraftätern. Baden-Baden: Nomos, S. 139-151.

Hammerschlag H, Schwarz 0 (1998) Das Gesetz zur Bekämpfung von Sexualdelikten und anderen gefährlichen Straftaten - ein erster Überblick. NStZ 18: 321-326.

Hendriks I, Bullens R (1998) Dauerhafte Veränderung - I. Handbuch Rückfallvorbeugung. Leiden: Ambulant Bureau Jeugdwelzijn zarg.

Hilpert H, Schwarz R, Beese F (Hrsg.) (1981) Psychotherapie in der Klinik - Von der therapeutischen Gemeinschaft zur stationären Psychotherapie. Heidelberg: Springer.

Krampen G (1991) Fragebogen zu Kompetenz- und Kontrollüberzeugungen (FKK). Göttingen: Hogrefe.

Layne T (1994) Die therapeutische Arbeit mit den Tätern. In: Gegenfurtner M, Bartsch B (Hrsg.) Sexueller Missbrauch von Kindern und Jugendlichen. Hilfe für Täter und Kind. Magdeburg: Westarp, S. 118-130.

Madanes C (1997) Sex, Liebe und Gewalt. Heidelberg: Carl-Auer-Systeme.

Müller-Isberner R, Thomas V (1992) Psychotherapie von Sexualstraftätern im Maßregelvollzug. Recht u. Psychiatrie 10: 42-47.

O'Callaghan D, Print B (1994) Adolescent Sexual Abusers. Research, Assessment and Treatment. In: Morrison T (Hrsg.) Sexual Offending against Children. London: Routledge.

Rehder U (1993) Sexuell abweichendes Verhalten - Klassifikation, Ursachen, Behandlung. In: Egg R (Hrsg.) Sozialtherapie in der goer Jahren. Wiesbaden: Kriminologische Zentralstelle.

Rösler M (1997) Die Prognose der Sexualdelinquenz bei Jugendlichen und Heranwachsenden. In: Warnke A, Trott GE, Remschmidt H (Hrsg.) Forensische Kinder- und Jugendpsychiatrie. Bern: Huber.

Rotthaus KP (1998) Neue Aufgaben für den Strafvollzug bei der Bekämpfung von Sexualdelikten und anderen gefährlichen Straften. NStZ: 597-600.

Rotthaus W (1990) Stationäre systemische Kinder- und Jugendpsychiatrie. Dortmund: moderenes lernen.

Rotthaus W (1998) Freiwilligkeit und Zwang und was dazwischen liegt - Ein Plädoyer für variable Therapiekonzepte. In: Wagner E, Werdenich W (Hrsg.) Forensische Psychotherapie. Wien: Facultas, S. 271-280.

Rotthaus W, Oruber T (1997) Systemische Tätertherapie mit Jugendlichen und Heranwachsenden. Einladung zur Konstruktion einer Welt der Verantwortlichkeit. In: Amann G, Wipplinger R (Hrsg.) Sexueller Missbrauch: Überblick zu Forschung, Beratung und Therapie. Ein Handbuch. Tübingen: dvgt, S. 573-585.

Russinger U, Wagner E (1999) Gewalt - Zwang - System. Systemisch-konstruktivistische Konzepte in institutionellen Zwangskontexten. Z System Ther 17: 144-156.

Schall H, Schreibauer M (1997) Prognose und Rückfall bei Sexualstraftätern. NJW: 2412-2420.

Schmidt G (1996) Inhaftierte Sexualstraftäter. Bewährungshilfe 1: 3-16.

Schüler-Springorum H et al. (1996) Sexualstraftäter im Maßregelvollzug - Grundfragen ihrer therapeutischen Behandlung und der Sicherheit der Allgemeinheit - Gutachten der unabhängigen Expertenkommission. Mschr Krim 3: 147-209.

Thomas-März V, Müller-Isberner R (1995) Psychotherapie mit Sexualstraftätern. ZfStrVo 44: 344-347.

Wiederholt IC (1989) Psychiatrisches Behandlungsprogramm für Sexualstraftäter in der Justizvollzugsanstalt München. Zf'StrVo 38: 231-236. 